\title{
Soft gamma repeaters activity in time
}

\author{
S.B. Popov ${ }^{1, \star}$ \\ Sternberg Astronomical Institute, Universitetski pr. 13, Moscow 119991, Russia
}

The dates of receipt and acceptance should be inserted later

Key words gamma rays: bursts — stars: neutron

In this short note I discuss the hypothesis that bursting activity of magnetars evolves in time analogously to the glitching activity of normal radio pulsars (i.e. sources are more active at smaller ages), and that the increase of the burst rate follows one of the laws established for glitching radio pulsars. If the activity of soft gamma repeaters decreases in time in the way similar to the evolution of core-quake glitches $\left(\propto t^{5 / 2}\right)$, then it is more probable to find the youngest soft gamma repeaters, but the energy of giant flares from these sources should be smaller than observed $10^{44}-10^{46}$ ergs as the total energy stored in a magnetar's magnetic field is not enough to support thousands of bursts similar to the prototype 5 March 1979 flare.

(c) 0000 WILEY-VCH Verlag GmbH \& Co. KGaA, Weinheim

\section{Introduction}

Soft gamma repeaters (SGRs) are known to demonstrate three main types of activity: weak, intermediate, and giant flares (see a recent review in Woods \& Thompson 2006). 1 More energetic events are less frequent than weak bursts. The distribution of SGRs burst energy has a power-law shape, $d N / d E \propto E^{-\alpha}, \alpha \sim 5 / 3$. This distribution is similar to the Gutenberg-Richter law established for terrestrial quakes (Cheng et al. 1996; Göğüş et al. 1999). A lot is known about properties of bursts of different kinds, but the evolution of the rate of bursts during lifetime of SGRs in not known due to poor statistics and relatively short period of exploration of these sources.

Here I discuss a possible link between SGRs bursts and period glitches, especially glitches driven by starquakes. Such connection was suggested long ago. Also the possibility that starquakes can power cosmic gamma-ray bursts was discussed by Blaes et al. (1989) (see references to earlier papers therein). However, to the author's knowledge, the possible similarity between the evolution of the bursts rate during a SGR lifetime and the evolution of the rate of glitches derived from radio pulsar observations has never been discussed in literature. The reason lies in the accepted difference between radio pulsar and magnetar glitches. In the case of radio pulsars, two types of glitches are distinguished: Vela-like and Crab-like. They are thought to be due to either starquakes or superfluid vortex unpinning. Glitches in magnetars are believed to be connected with strong magnetic fields of these stars. Nevertheless, I think that a discussion of possible similarities between the evolution of glitch

\footnotetext{
^ Corresponding author: e-mail: polar@sai.msu.ru

${ }^{1}$ Here often I do not discuss separately, in the context of the proposed hypothesis, hyperflares (HFs) like the one from SGR 1806-20 observed on 2004 Dec 27. Such events are just included into the class of giant flares.
}

rates in different kinds of neutron stars (NSs) is worth while, especially in connection with giant flares.

During $\sim 25-30$ years of observation four strong flares have been detected from four known SGRs (Mazets et al. 2004) 2 . Despite their smaller rate, due to large energy release these bursts dominate among other types of bursting activities of SGRs. The rate of such flares was usually assumed to be $\sim 0.02-0.01$ per year per source (Woods $\&$ Thompson 2006) based on just two giant flares (GFs) (5 March 1979 and 28 August 1998). However, with inclusion of the 27 December 2004 flare and with inclusion of the 18 June 1998 burst of SGR 1627-41 the rate becomes higher. Still, below I shall use a conservative estimate 0.02 GFs per year per source. Data on possible extragalactic SGR flares (Ofek 2007) is only marginally compatible with this rate, and I briefly discuss this problem below.

Previously several authors (Nakar et al. 2006; Popov \& Stern 2006; Lazzati, Ghirlanda \& Ghisellini 2005; Ofek 2007) addressed the problem of extragalactic SGR flares. They used different archive data (BATSE, IPN) to search for examples of extragalactic GFs and HFs, and to put limits on the fraction of SGR flares among short GRBs (see Nakar 2007 for a review on short GRBs and for some discussion of the fraction of SGR flares among them). Estimates show that GFs can be observed by most of the detectors (from BATSE to SWIFT) up to distances of few Mpc (Palmer et al. 2005). I.e., even SWIFT cannot observe GFs from most

\footnotetext{
2 Often only two bursts - 05 March 1979 from SGR 0526-66 and 27 August 1998 from SGR 1900+14 - are called giant bursts. Many authors do not include the flare from SGR 1627-41 on 18 June 1998 into the list of giant flares, as for this event a pulsating "tail" was not observed. The flare of SGR 1806-20 is also sometimes classified as an event of a different type - a hyperflare - HF.
} 
of the Virgo cluster - only HFs can be detected from such distance (Hurley et al. 2005). 3

Usualy authors apply the value of GFs rate $\sim 0.02-$ 0.01 per year per source to the whole life period of SGRs, i.e. the rate of GFs is assumed to be constant in time. In this short note I discuss an alternative assumption. NS activity of different kinds usually decreases with time, and the GF rate should not be an exception. Possibly the closest analogue is the evolution of glitch (especially, starquake) rate.

\section{Possible evolution of the giant flare rate during SGRs lifetime}

Bursting activity of magnetars is known to be associated with glitches. Such an association naturally arose already in the early papers by Thompson \& Duncan (1996). For SGRs themselves, period determinations are not precise enough to allow glitch detection 4 , but for anomalous X-ray pulsars (AXPs) such correlation between bursts and glitches has been found. For example, the source 1E2259+586 showed a glitch coincident with a burst (Kaspi et al. 2003; Woods et al. 2006).

Despite the possibility that glitches of SGRs and AXPs can be different from normal radio pulsar glitches due to the role of superstrong magnetic fields, here I want to propose the following hypothesis: bursting activity of magnetars (mainly the GF rate) evolves with time similarly to the glitching activity of normal radio pulsars.

\footnotetext{
3 Details about SWIFT sensitivity can be found in (Band 2006). SWIFT appears to be more sensitive than BATSE to long bursts, but not for short hard events. The advantage of SWIFT is in better localization of short events. BAT onboard SWIFT is less sensitive than BATSE to energies $>100 \mathrm{keV}$. HFs are relatively hard events: spectra of the HF 27 December 2004 can be described as a blackbody with $\mathrm{T} \sim 200 \mathrm{keV}$. So, in general, SWIFT is not much better than BATSE for detection of bursts like the only known HF. Other experiments, like BeppoSAX and HETE-II, also are not more sensitive than BATSE to short hard bursts. However, as noted by Band (2006), an analysis of short-duration triggers by SWIFT which do not result in successful image triggers can result in discovery of more short flares. Up to the limiting distance for SWIFT detection the extrapolation of the galactic rate of GFs gives an estimate of few events in a year, so SWIFT with its sky coverage of about 1.4 steradians can detect approximately one GF per year. HFs can be detected from larger distances: 30-40 Mpc by BATSE, and up to $70 \mathrm{Mpc}-$ by SWIFT (Hurley et al. 2005). Scaling the galactic rate of HFs - one in 30 years - Hurley et al. (2005) gives the rate about 50 per year for SWIFT. However, any rescaling of the galactic rate of HFs is extremely uncertain as only one event has been observed. Results presented in (Popov \& Stern 2006; Lazzati et al. 2005; Ofek 2007) show that the rate of extragalactic HFs is much lower than that expected from a naive scaling (based on starformation rate or supernova rate, etc.) given one observed event from SGR 1806-20 in our Galaxy. Upper limits from non-detection of HFs from the Virgo cluster by BATSE provide an estimate $\sim 10^{-3} \mathrm{yr}^{-1}$ per Milky Way-like galaxy (Popov \& Stern 2006). This number after rescaling again gives an estimate of about one detection per year for SWIFT. Still, larger statistics of HFs detection is necessary. Recent observations (Frederiks et al. 2007; Ofek et al. 2006) have already provided a candidate for a HF in a close-by galaxy with high starformation rate (such stellar systems are the most prominent hosts of GFs and HFs). Hopefully, more events can be detected by SWIFT during its lifetime.

${ }_{4}$ Also glitches can be masked by a transient period increase as the one observed for SGR 1900+14, see discussion, for example, in Thompson, Lyutikov \& Kulkarni (2002).
}

Glitches of radio pulsars are well known since 1969 (Radhakrishnan \& Manchester 1969; Reichley \& Downs 1969). Relatively recent data on statistical properties of glitches can be found in Lyne, Shemar \& Graham Smith (2000). The most up-to-date information is available on the Web in the ATNF catalogue 5 .

Long-period pulsars also show glitches. The wellknown long period glitching pulsar is PSR B0525+21. It has $p=3.75 \mathrm{~s}, \dot{p} \approx 410^{-14}, B=1.2410^{13} \mathrm{G}$, age $\sim 1.510^{6}$ yrs. Recently, the glitch was reported (Janssen \& Stappers 2006) from PSR J1814-1744 with $p \sim 4 \mathrm{~s}$, $B \sim 5.510^{13} \mathrm{G}, \dot{p} \sim 7.410^{-13}$, and age $\sim 8.510^{4} \mathrm{yrs}$. So, probably, magnetars which have somehow similar periods can also suffer normal (i.e. not magnetically driven) glitches. It is interesting to note, that as there are claims that SGRs are born from the most massive stars which still can produce NSs (Figer et al. 2005; Gaensler et al. 2005), then for them it is possible to apply the consideration (see, for example, Alpar \& Ho 1983) that more massive NSs can produce more rare, strong glitches. This becomes posible due to a solid core formation if a very stiff equation of state is realized in nature. Formation of a solid core necessary to produce very large glitches can be an interesting option distinguishing between SGRs and "normal" NSs.

Recently, a glitch was proposed to interpret the behavior of one of the Magnificent Seven - radioquiet close-by cooling NSs - RX J0720-3125 (van Kerkwijk et al. 2007). It is a NS with the spin period $8.4 \mathrm{~s}$. If the glitch of RX J0720-3125 is due to unpinning, then using standard formulae (Alpar \& Baykal 1994) it is possible to estimate the reccurence time of glitches in this source. Surprisingly, the time is about 10 years. So, it is quite probable to observe one since 1997 So, even for long-period NSs which do not show radio pulsar behaviour glitches can be a normal phenomena.

Two main models of glitches are discussed: starquakes (Ruderman 1969) and vortex unpinning (Anderson \& Itoh 1975). The first of these two models can be especially good analogue of magnetar glitches. Let us discuss the evolution of glitches rate according to these two well-known models.

For the model of unpinning of superfluid vortices Alpar \& Baykal (1994) demonstrated arguments in favour of the following glitch property: for all NSs the ratio $\delta \Omega / \Omega$ is nearly the same. Where $\Omega$ is a spin frequency, and $\delta \Omega$ is the parameter which determines the time interval between glitches according to the following simple relation:

$t_{\mathrm{g}}=\frac{\delta \Omega}{\dot{\Omega}}$.

Parameter $\delta \Omega$ is the critical value of the difference between the rotation frequencies of normal matter and the su-

\footnotetext{
5 http://wwwatnf.atnf.csiro.au/research/pulsar/psrcat/

${ }^{6}$ In this source glitches, probably, generate Tkachenko waves (Tkachenko 1966, Ruderman 1970), which in their turn lead to precession.
} 
perfluid at a boundary layer. $\delta \Omega$ itself can be estimated as (Alpar \& Baykal 1994; Cheng \& Chi 1996):

$\delta \Omega=\Delta \Omega \frac{I_{0}}{I_{\mathrm{p}}}$,

here $I_{\mathrm{p}}$ is the effective moment of inertia of the region of a pinning layer.

Combining these two formulae we obtain the relation for the time between glitches:

$t_{\mathrm{g}}=\frac{2 I_{0}}{I_{\mathrm{p}}} \frac{\Delta \Omega}{\Omega} t \propto t$,

where $t$ is the age of a pulsar, $t=\Omega / 2 \dot{\Omega}$. To obtain the proportionality $t_{\mathrm{g}} \propto t$ it is assumed that $\Delta \Omega / \Omega$ is constant during a NS lifetime.

If for the age $t_{0}=1000-2000 \mathrm{yrs}$ a SGR has GF rate equal to one burst in $\sim 25-50$ years, then for the age of about 100 yrs the rate is about one burst in 1-5 years. It is unclear whether a NS can produce bursts at earlier time since only at the age 50-100 yrs it becomes isothermal (Prakash et al. 2001).

However, eq. 1 is valid for the superfluid vortex unpinning model (Alpar \& Baykal 1994), which is unlikely to be valid for magnetars. For the core-quake model, the formula for $t_{\mathrm{g}}$ is different (Alpar \& Ho 1983; Alpar \& Baykal 1994):

$t_{\mathrm{g}}=\frac{2(A+B) \phi \Delta \Omega / \Omega}{I_{0} \Omega \dot{\Omega}}$.

This scenario gives a very quick rise of the glitch rate towards the pulsar's youth:

$t_{\mathrm{g}} \propto t^{5 / 2}$,

if $A, B, \phi$, and $\Delta \Omega$ are constant. If $\Delta \Omega$ decreases with time, then the dependence is slightly less steep. For example, for $\Delta \Omega / \Omega=$ const we obtain $t_{\mathrm{g}} \propto t^{2}$. Therefore, if the rate of GFs follows the same law (for example, glitches can trigger GFs), then young SGRs produce GFs much more often that the observed 1000-year old sources. Magnetic field decay can lead to a dependence of the glitching period on time even steeper than that in eq. 5 .

In section 3 I briefly discuss some consequencies of this hypothesis.

\section{$2.1 \Delta \Omega / \Omega$ estimate}

Using eqs. 3 or 4 it is possible to obtain a naive estimate of the value $\Delta \Omega / \Omega$ after a GF. Time intervals between GFs are about 50 years. In the case of eq. 3 assuming $I_{\mathrm{p}}=10^{-2} I_{0}$ (Alpar \& Baykal 1994; Cheng \& Chi 1996) we obtain $\Delta \Omega / \Omega \sim 10^{-4}$. This is a very large value.

In the case of eq. 4 for $A=10^{52}$ erg, $\phi=10^{-3}, B=$ $10^{48} \mathrm{erg}, \Omega \sim 1 \mathrm{rad} \mathrm{s}^{-1}, \dot{\Omega} \sim 10^{-11} \mathrm{rad} \mathrm{s}^{-2}$, and $I_{0} \sim$ $10^{45} \mathrm{~g} \mathrm{~cm}^{2}$ we obtain $\Delta \Omega / \Omega \sim 10^{-6}$. Characteristics of the glitch of $1 \mathrm{E} 2259+586$ are consistent with this estimate by Kaspi et al. (2003). Of course, the use of parameters similar to radio pulsars is an oversimplification. For starquakes, in the case of magnetars $\Delta \Omega / \Omega$ hardly can be that high as it should be limited by oblateness: $\epsilon \sim \Delta \Omega / \Omega$ and $\epsilon \sim a / g \sim$ few $10^{-9} \Omega^{2}$, where $g$ is the gravitational acceleration and $a$ is the centrifugal one. Smaller $\Delta \Omega / \Omega$ correspond to smaller $t_{\mathrm{g}}$. For realistic $\Delta \Omega / \Omega$ and other parameters as specified above glitches should be more frequent. This can mean that not every glitch lead to a GF.

\section{Discussion}

\subsection{Detectability of SGRs of different age}

Known SGRs have spin-down ages of about 1-2 thousand years (Woods \& Thompson 2006). It has been claimed that observations of younger SGRs are less probable due to a smaller number of such objects. However, if our hypothesis is correct it is not so! If the bursting activity is scaled in linear proportion to a source's age, then there is an equal probalitity to find a SGR of any age. If $t_{\mathrm{g}}$ inreases with time faster than it is described in eq. 3 then the probability to detect a younger SGR is even higher, despite that the number of such sources is small. This means that the probability of detection is biased towards younger objects.

The fact that we do not detect younger SGRs in our Galaxy (and in near-by galaxies like Magellanic clouds) tells us, that there are no younger sources of this type in our close proximity, as they should burst more often than the four known sources, and in our vicinity we cannot miss even weak SGR flares. In the case of eq.5it is especially obvious as the increase of the burst rate for young sources is extremely strong. Or, one can turn the argument around, the absence of other known close-by SGRs can rule out significant decrease of flare rate in time, if the rate of formation of these sources in our neighborhood is larger than one in several hundred years (on the formation rate of magnetars see the recent paper by Gill \& Heyl 2007).

If activity of SGRs decays with time, then bounds obtained in (Popov \& Stern 2006; Lazzati et al. 2005; Ofek 2007 ) become stronger, as the total number of bursts of any kind is larger than the one derived by naive use of the observed (from the four sources) rate in the form of a constant value valid for the whole lifetime of a magnetar, unless GFs and HFs from younger SGRs are somehow weaker.

\subsection{Powerful flares of young SGRs and energy crisis}

There are suggestions (see, for example, Hurley et al. 2005) that younger magnetars can produce flares even more powerful than the one observed on December 27, 2004. This suggestion is based on the fact that young SGRs must have shorter spin periods and higher magnetic fields. If so, then we can expect to find such young SGRs in starforming galaxies (Popov 2005; Popov \& Stern 2006), and possibly two candidates have recently been observed (Frederiks et al. 2006; Ofek et al. 2006; Golenetskii et al. 2007). However, if bursts of young magnetars appear much more often, then 
it is necessary to estimate whether there is enough energy resources for the increased rate of more powerful flares. It is important to note here, that as weak bursts seem to cluster around more energetic ones we do not expect to observe just an increase of weak bursts activity without corresponding increase in the number of GFs, and, vice versa, an increase of only GFs rate is unexpected, too.

Suppose that the formula 3 is valid. Then with the present-day rate of GFs of about one in 50 yrs per source, we can expect, that a NS in the time interval $100<t<2000$ can produce about 100 GFs. The total energy stored in the magnetic field can be estimated as:

$E_{\text {tot }}=210^{47} B_{15}^{2}$ erg.

So, there is enough energy to produce $100 \mathrm{GFs}$ with energy of $\sim 10^{45} \mathrm{erg}$. However, there is not enough energy to produce significantly more powerful flares in a SGR youth. To produce a comparable amount of more energetic flares it is necessary to have initial field of about $10^{16} \mathrm{G}$ (Stella et al. 2005). This is possible if young magnetars have much higher magnetic fields than the observed ones (see, Pons \& Geppert 2007).

As supernova rates in galaxies with extreme starformation can be up to 100 times higher than that in the Milky Way, in such systems we can expect several SGRs with ages of about 100 years. Then the rate of GFs from such "supernova factories" can be one in few months. Non-detection of such bursts or at least small number of candidates coincident with expectations of random projections (Popov 2005) can be an additional argument against frequent HFs from young SGRs.

In the case of viability of the eq. 5 the situation is different. We can expect $>10^{3} \mathrm{GFs}$ during the interval $100<t<2000$ years. In this case, even for constant energies of flares we face an energy crisis. For an increase of a flare energy towards smaller ages the crisis becomes even more dramatic. The energy of a glitch itself is too low to contribute significantly to a GF:

$E=I_{0} \Omega \Delta \Omega=10^{40} \Omega_{1}^{2}\left(\frac{\Delta \Omega}{\Omega}\right)_{-5} \mathrm{erg}$,

here $\left(\frac{\Delta \Omega}{\Omega}\right)_{-5}=(\Delta \Omega / \Omega) / 10^{-5}$. In the case of magnetars $\Delta \Omega / \Omega$ after a starquake can not be that big as it is limited by oblateness that is small for SGRs spin periods, $\Delta \epsilon \leq \epsilon \sim$ few $10^{-9} \Omega^{2}$. However, estimates of oblateness for RX J0720.4-3125 (one of the so-called Magnificent Seven) is $\sim 410^{-8}$ (Haberl 2007). Even higher values of deformation can be reached due to strong toroidal field $B_{\mathrm{t}}$. Stella et al. (2005) showed that the value of ellipticity can be as high as $\sim 6.410^{-4} B_{\mathrm{t}, 16.3}^{2}$. However, this ellipticity can be unrelated to glitches.

Thermal energy of a NS after few hundred years is not enough to support a magnetar activity. The total rotational energy is also unsufficient to support significant number of GFs when a magnetar slowed down: $E_{\text {rot }} \approx 10^{45} I_{45} \Omega^{2}$.
So, if the rate of GFs follows the same decay with time as the rate of radio pulsar glitches in the starquake model, an avegare energy of GFs of young SGRs should be smaller in comparison with older objects in order not to face the energy crisis. These considereations can be important for estimates of detectability of extragalactic GFs (see discussion of this subject in Duncan 2001; Popov \& Stern 2006; Lazzati et al. 2005, Crider 2006; Ofek 2007 and references therein).

Let the present day typical energy in a giant flare be $E_{0}$, and the interval between such flares $k_{0}$. Let them vary as: $k_{0}\left(t / t_{0}\right)^{a}$ and $E_{0}\left(t / t_{0}\right)^{b}$. Naively one can expect $a>0$ and $b<0$. Then the total emitted energy is:

$E_{\mathrm{em}}=\int_{t_{1}}^{t_{2}} \frac{E_{0}\left(t / t_{0}\right)^{b}}{k_{0}\left(t / t_{0}\right)^{a}} d t$.

For $b=0$ (i.e. constant flare energy) the energy crisis appears for $a \sim 2.5$ for $k_{0}=50$ yrs and $E_{0}=310^{44} \mathrm{erg}$. For $b<0$ the crisis appears even for smaller $a$.

It is important to note, that the evolution of the giant bursts rate cannot solve the problem of the deficit of events in the direction of the Virgo cluster. The contradiction between galactic and extragalactic rates discussed in (Popov \& Stern 2006) is based on the observed rate of GF in our Galaxy and its rescaling with the supernova rate. Smaller number of energetic HFs from very young magnetars cannot change the total rate significantly.

\subsection{SGRs - ultrahigh energy phenomena - starformation galaxies}

Eichler (2005) recently proposed (see also Singh, Ma \& Arons 2004) that GFs can be an important source of ultrahigh energy particles. In particular, the author discussed a possibility of ultrahigh energy cosmic rays (UHECR) generation during these bursts. Increased bursting activity of young SGRs can be responsible for even higher contribution to UHECR production. In this respect it is interesting to remember the result by Giller, Michalak \& Smialkowski (2003) These authors claim that there are correlations between UHECR and ultra-luminous IR galaxies (ULIRGs). In particular, these authors discussed the galaxies Arp 299 and NGC 3256. The same objects were also suggested by us (Popov 2005; Popov \& Stern 2006) as good candidates to host multiple young SGRs. I would like to underline a possible role of very young magnetars, mainly populating starforming regions, in a hypothetical UHECR production, and their strong link with galaxies with high starformation rate, especially with ULIRGs. If the rate of energy release is larger for young SGRs then these sources can contribute to the production of energetic particle in starforming galaxies on the level higher than estimated from the known galactic population of SGRs, i.e. naive scaling based just on the number of SGRs is not valid. Still, the total contribution of magnetars into the UHECR production is anyway limited by the total energy stored in their magnetic fields. 


\section{Conclusions}

I propose and discuss the hypothesis that the bursting activity of SGRs decreases with time similar to the glitching activity of normal radio pulsars. This assumption leads to several interesting consequences. Namely,

- For the same burst energy the probability to detect a SGR at least does not diminish with decreasing SGR age. Even it can be more probable to detect young SGRs due to their more frequent bursts. For example, the galactic (in general, Local group) population of SGRs should already include all the youngest objects of this type.

- If the bursting activity of magnetars rapidly decreases with time, but a typical GF energy is constant (or decreases too), then we face an energy crisis: there is not enough energy in the magnetic field to support thousands of flares similar to, say, the 5 March 1979 burst.

- Young SGRs should burst more often, but their giant flares should be less powerful than the known ones.

Absence of many identified extragalactic giant and hyper flares by itself can rule out joint decrease of the flare rate and flare energy with time.

\section{Acknowledgments}

I want to thank Drs. V.A. Belokurov, M. Lyutikov, M.E. Prokhorov and B.E. Stern for comments. The work was supported by the RFBR grant 06-02-16025 and by INTAS.

\section{References}

Alpar, M.A., Ho, C.: 1983, MNRAS 204, 655

Alpar, M.A., Baykal, A.: 1994, MNRAS 269, 849

Anderson, P.W., Itoh, N.: 1975, Nature 256, 25

Band, D.L.: 2006, ApJ 644, 378

Blaes, O., Blandford, R., Goldreich, P., Madau, P.: 1989, ApJ 343, 839

Cheng, K.S., Chi, X.: 1996, A\&A 306, 326

Cheng, B., Epstein, R.I., Guyer, R.A., Young, A.C.: 1996, Nature 382,518

Crider, A.: 2006, in: "Gamma-Ray Bursts in the Swift Era", Eds. S.S. Holt, N. Gehrels, and J.A. Nousek. AIP Conference Proceedings, Vol. 836. Melville, NY: American Institute of Physics, p.64 [ArXiv: astro-ph/0601019]

Duncan, R.: 1998, ApJ 498, L45

Duncan, R.C.: 2001, in Proc. 20th Texas Symposium on relativistic astrophysics, AIP conference proceedings, Vol. 586. Eds. J. Craig Wheeler and H. Martel, p. 495 [ArXiv: astro-ph/0103235]

Eichler, D.: 2005, astro-ph/0504452

Figer, D.F., Najarro, F., Geballe, T.R., Blum, R.D., Kudritzki, R.P.: 2005, ApJ 622, L49

Frederiks D. D., Palshin, V. D., Aptekar, R. L. et al.: 2007, Astronomy Letters 33, 19 [ArXiv: astro-ph/0609544]

Gaensler, B.M., McClure-Griffiths, N.M., Oey, M.S., Haverkorn, M., Dickey, J.M., Green, A.J.: 2005, ApJ 620, L95
Gill, R., Heyl, J.: 2007, MNRAS in press [ArXiv: astro-ph/0703346]

Giller, M., Michalak, W., Smialkowski, A.: 2003, in: Proceedings of the 28th ICRC, Tsukuba, Japan, p.727 [ArXiv: astro-ph/0308532]

Göğüs, E., Woods, P.M., Kouveliotou, C. et al.: 1999, ApJ, 526, 93

Golenetskii, S., Aptekar, R. Mazets, E. et al.: 2007, GCN 6088

Haberl, F.: 2007, Ap\&SS 308, 181

Hurley, K., Boggs, S.E., Smith, D.M. et al.: 2005, Nature 434, 1098

Janssen, G.H., Stappers, B.W.: 2006, A\&A 457, 611

Kaspi, V., Gavriil, F.P., Woods, P.M., Jensen, J.B., Roberts, M.S.E., Chakrabarty, D.: 2003, ApJ 588, L93

Lazzati, D., Ghirlanda, G., Ghisellini, G.: 2005, MNRAS 361, L8

Lyne, A.G., Shemar, S.L., Graham Smith, F.: 2000, MNRAS 315, 534

Mazets, E.P., Golenetskii, S., Aptekar, R., Frederiks, D., Pal'shin, V., Cline, T.: 2004, GCN 2922

Nakar, E., Gal-Yam, A., Piran, T., Fox, D.B.: 2006, ApJ 640, 849

Nakar, E.: 2007, Physics Reports 442, 166

Ofek, E.O. Kulkarni, S.R., Nakar, E. et al.: 2006, ApJ 652, 507

Ofek, E.: 2007, ApJ 659, 339

Palmer D.M. et al.: 2005, Nature 434, 1107

Pons, J., Geppert, U.: 2007, A\&A 470, 303

Popov, S.B.: 2005, ArXiv: astro-ph/0502391

Popov, S.B., Stern, B.E.: 2006, MNRAS 365, 885

Prakash, M., Lattimer, J.M., Pons, J.A., Steiner, A.W., Reddy, S.: 2001, in: "Physics of neutron star interiors", eds. Blaschke D., Glendenning N.K., Sedrakian A., p.364 [ArXiv: astro-ph/0012136

Radhakrishnan, V., Manchester, R.N.: 1969, Nature 222, 228

Reichley, P.E., Downs, G.S.: 1969, Nature 222, 229

Ruderman, M.: 1969, Nature 223, 597

Ruderman, M.: 1970, Nature 225, 619

Singh, S., Ma, C.-P., Arons, J.: 2004, Phys. Rev. D 69, 063003

Stella, L., Dall'Osso, S., Israel, G. L., Vecchio, A. : 2005, ApJ, 634, L165

Thompson, C., Duncan, R.: 1996, ApJ 543, 322

Thompson, C., Lyutikov, M., Kulkarni, S.R.: 2002, ApJ 574, 332

Tkachenko, V.K.: 1966, Zh. Exp. Theor. Fiz. 50, 1573 [English translation: Sov. Phys. JETP 23, 1049]

van Kerkwijk, M.H., Kaplan, D.L.. Pavlov, G.G., Mori, K.: 2007, ApJ 659, L149

Woods, P.M., Kaspi, V.M., Thompson, C. et al.: 2004, ApJ 605, 378

Woods, P.M., Thompson, C.: 2006, In: “Compact stellar X-ray sources", Eds. W. Lewin and M. van der Klis, Cambridge Astrophysics Series 39, 547 [ArXiv: astro-ph/0406133] 